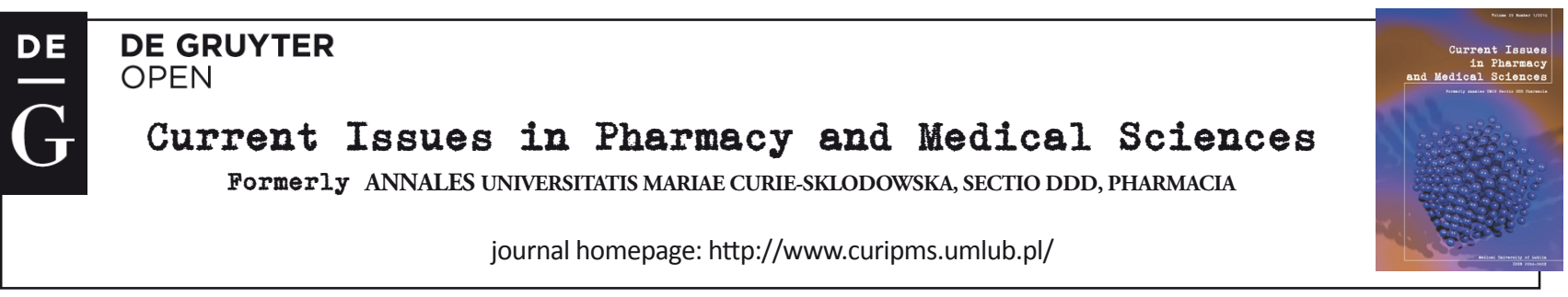

\title{
Contemporary pharmacological obesity treatments
}

\author{
Katarzyna Kaszubska, Barbara Budzynska*, Grazyna Biala \\ Department of Pharmacology and Pharmacodynamics, Medical University of Lublin, 4a Chodzki, 20-093 Lublin, Poland
}

\begin{tabular}{|c|c|}
\hline ARTICLE INFO & ABSTRACT \\
\hline $\begin{array}{l}\text { Received } 08 \text { February } 2016 \\
\text { Accepted } 14 \text { March } 2016\end{array}$ & $\begin{array}{l}\text { In the last few years, obesity has become a global epidemic. Consequently, worldwide } \\
\text { costs associated with managing obesity and obesity-related comorbidities are huge. }\end{array}$ \\
\hline $\begin{array}{l}\text { Keywords: } \\
\text { pharmacotherapy, } \\
\text { obesity, } \\
\text { Body Mass Index, } \\
\text { drug. }\end{array}$ & $\begin{array}{l}\text { Numerous studies have focused on discerning the appropriate proper treatment of weight } \\
\text { related problems such as overweight and obesity. Moreover, many clinical trials have been } \\
\text { conducted for many years in order to introduce effective anti-obesity drugs. The aim } \\
\text { of the present review is to provide an overview of current and future pharmacotherapy } \\
\text { for obesity, and to provide the reader with a determination of the concentration and } \\
\text { composition of long and short term anti-obesity drugs, doing so by placing emphasis } \\
\text { on pharmacotherapy and up-to-day solutions. It should be noted that, currently, the } \\
\text { worldwide pharmacotherapy is represented by phendimetrazine, benzphetamine and } \\
\text { diethylpropion, as well as by orlistat, lorcaserin, phentermine/topiramate, naltrexone/ } \\
\text { bupropion and liraglutide. In our paper, individual cases of patients' needs are thoroughly } \\
\text { illustrated by way of examples. Medical prescriptions and contraindications are also } \\
\text { described. }\end{array}$ \\
\hline
\end{tabular}

\section{INTRODUCTION}

During the last few decades, the environmental situation, food supply, human health situations, technology and medicine have changed a lot due to the global epidemic of obesity. Every single nation and economic status is affected by this epidemic. Indeed, every third person in the world is either obese or overweight.

Many health problems are closely connected with weight, for example: cardiovascular disease, type 2 diabetes mellitus, cancer, dementia and osteoarthritis [9]. Yet, what does it mean to be obese or overweight? The term "obesity" means the existence of a condition which is characterized by the excessive accumulation and storage of fat in the body. The word "obesity", however, may be mistaken with the term "overweight". The latter term is defined as the presence of an abnormal accumulation of body fat, usually $20 \%$ or more, over an individual's ideal body weight. It is worth mentioning that these two terms do not have the same meaning. This is by virtue of the fact that obesity comes about in time, when a body does not use the amount of received calories. Indeed, obese persons can accumulate so much body fat that this situation might have a negative effect on their health. If the Body Mass Index (BMI) is between 25 and $29.9 \mathrm{~kg} / \mathrm{m}^{2}$,

\footnotetext{
* Corresponding author

e-mail: basia.budzynska@umlub.pl
}

a patient is considered to be overweight. If the BMI is between $30 \mathrm{~kg} / \mathrm{m}^{2}$ or over, the patient is considered obese. BMI between: 30.0-34.9 is considered as a class I obesity, 35.0-39.9 - a class II obesity, and $\geq 40.0$ - a class III obesity [5].

In accordance with a 2014 survey, the World Health Organization (WHO) estimates that more than 1.9 billion adults (39\%) worldwide are overweight and over 600 million adults $(13 \%)$ are obese [14]. To add to this, the National Health and Nutrition Examination Survey of 2011-2012, has assessed that over two-thirds $(68.5 \%)$ of all adults in the US were overweight or obese, with $34.9 \%$ classified as obese and $6.4 \%$ classified as extremely obese [14]. When it comes to European Union countries, the prevalence of obesity has doubled among both men and women. According to WHO, as of 2008 , more than $50 \%$ of all men and women in the European Region were overweight, and about $23 \%$ of all women and $20 \%$ of all men were obese [14]. Moreover, the Central Statistical Office has reported that, as of 2014 , more than $62 \%$ of all men in Poland weigh too much (44\% were overweight and $18 \%$ were classified as obese). Furthermore, among women, almost $46 \%$ of all women weigh too much (30\% were overweight, and $16 \%$ were obese) [4]. The alarming fact is that obesity also increasingly applies to children around the world [8]. 


\section{CAUSES OF OBESITY}

First of all, weight problems are linked with the unhealthy diets and increased caloric intake that tip the energy balance equilibrium. Secondly, the reason for obesity coming about, is the practicing of a sedentary lifestyle which slows down the metabolism. Not sleeping enough may be counted as a third reason. Indeed, it has been demonstrated that sleep deprivation significantly disorders the biological body's functions, and this doubles the risk of being obese. Obesity can also be induced by malnutrition as a fetus, damage to the hypothalamus and by endocrinopathies. The last but not least factor of the overweight condition occurring is that of a faulty gene affecting the fat mass and obesity-associated protein known as alpha-ketoglutarate-dependent dioxygenase (FTO). Humans with a developed FTO gene tend to overeat fatty and high-energy foods without reaching the filling of satiety [6].

\section{COMPLICATIONS}

Obesity should be considered as a particular cause for concern by virtue of obesity increasing the risk of cardiometabolic disease, dementia, kidney disease, cancer, respiratory disease and osteoarthritis. Various disorders such as dyslipidemia, stroke, nonalcoholic steatohepatitis, type 2 diabetes mellitus, as well as gallbladder disease, cancers and coronary heart disease complicate human lives and are also connected with obesity. This presents huge healthcare challenges for the future [1]. This XXI century epidemic make children, even infants, suffer. Overweight children are prone to struggle with type 2 diabetes and cardiovascular disease. Moreover, they will encounter mental disorders, lower self-esteem and peer disapproval [1].

\section{TREATMENT}

Researchers and health-care professionals have been working for a long time in order to create innovative obesity treatments. Without a shadow of a doubt, it is clear that the very first steps in losing weight are to undertake physical activity and to follow a healthy diet. Patients with weight problems are recommended to reduce the consumption of calories (at least $500 \mathrm{kcal}$ per day) and to exercise [9]. Doing so is considered the most patient-friendly way in losing redundant fat.

Medical practitioners and dietitians state that pharmacotherapy is one of most up-to-day and effective methods for alleviating obesity. However, such pharmacotherapy should be adjusted to the individual patient's needs after a proper diagnosis of the patient's particular reasons of his obesity.

The chronic management of obesity is currently undertaken by way of five pharmacological medications, two of which were approved in 2014. These pharmacological therapies are options to aid weight loss in patients that are obese or to aid those who are overweight and who are considered to have additional risk factors [2].

There are two distinctive types of anti-obesity pharmacotherapy. The first one is tailored for a short period of time (about 12 weeks). The second is considered to be long term and intensive. It is worth mentioning that the former does not demonstrate clinically meaningful weight loss, whereas the latter does help obese and overweight patients achieve clinically meaningful weight loss [9].

\section{SHORT-TERM PHARMACOTERAPHY}

The American Food and Drug Administration (FDA) has approved four short-term (around 12 weeks) noradrenergic agents which help in obesity management. The most frequently prescribed drug is phentermine. This is indicated for short-term use as an adjunctive therapy in patients with BMI $\geq 30 \mathrm{~kg} / \mathrm{m} 2$, and this agent suppresses appetite by inhibiting adrenaline re-uptake. Patients take phentermine orally once or twice per day. The dose ranges from 15 to $37.5 \mathrm{mg}$ daily. It should be underlined that people struggling with cardiovascular problems and hypertension are not allowed to take phentermine (even in small doses). The most general side effects are insomnia and dry mouth [9].

Phendimetrazine, benzphetamine and diethylpropion, like phentermine, are being qualified as short-term adjunctive treatments for obesity, as these act as appetite suppressants. However, all three drugs have adverse effects and contraindications similar to those of phentermine, and they also pose increased potential for abuse [15].

\section{LONG-TERM PHARMACOTERAPHY}

The following long-term anti-obesity pharmacotherapies are approved by FDA, and are available by prescription. These are orlistat, lorcaserin, phentermine combine with topiramate, liraglutide and a combination of naltrexone and bupropione [Fig. 1].

Orlistat (marketed as "Xenical") is considered to be a selective and potent inhibitor of pancreatic lipase, and its administration prevents the hydrolysis and absorption of triglycerides in the human intestine. This leads to the excretion of nearly $30 \%$ of ingested fat [15]. By virtue of the fact that this drug is not quickly and significantly absorbed, there is barely any systemic reaction. Patients are even able to purchase orlistat over-the counter. However, this form comes with a lower dose of chemical ingredients $-60 \mathrm{mg}$. The drug form available only by prescription is more efficient due to the fact that the dose is doubled $-120 \mathrm{mg}$. According to conducted studies, orlistat is efficient in generating body weight loss. What is more, a randomized study called "XENical in the Prevention of Diabetes in Obese Subjects" (XENDOS) was performed in order to evaluate the orlistat efficacy (120 $\mathrm{mg}$ ) in preventing type 2 diabetes mellitus (T2DM) in obese patients. The outcomes showed that patients with normal and impaired glucose tolerance who used orlistat, lost more weight, in comparison with patients who used a placebo [9]. Currently, orlistat is one of the most recommended anti-obesity drugs. This because it shows high tolerance, yet, side effects like gastric discomfort, oily spotting, fecal urgency, oily stool and fecal incontinence may also appear. For that reason, patients are recommended to supplement the drug with multivitamins which contain beta-carotene and the fat-soluble vitamins. Pregnant women, hypersensitive 
patients and those with chronic malabsorption syndrome are not allowed to use this drug [9].

In 2012, the FDA granted a license to lorcaserin ("Belviq") for use as a long-term anti-obesity drug. Lorcaserin is a serotonin type $2 \mathrm{C}$ receptor agonist. This drug contributes to weight loss since it regulates energy balance and satiety in the human body, by activating the $5-\mathrm{HT}_{2} \mathrm{C}$ receptors in the hypothalamus. The recommended dosage of lorcaserin is $10 \mathrm{mg}$, taken orally twice a day. Studies showed that patients who used the prescribed dosage in a full year's study, of this medicine, lost more weight than did placebo-treated patients $(5.8 \%$ vs. $2.2 \%$, p $<0.001)$, and this significant difference in weight loss was sustained throughout the consecutive year $(7.2 \%$ vs. $2.9 \%, \mathrm{p}<0.001)$ [1]. Additional studies have revealed that lorcaserin is well tolerated by most patients, yet a problem made evident is that long-term usage of the drug may cause valve abnormalities, despite its selectivity for $5-\mathrm{HT}_{2} \mathrm{C}$ receptors. Indeed, pooled echocardiographic data from 5249 trial participants indicated a non-statistically significant risk ratio of 1.16 (95\% confidence interval [CI] 0.81-1.67) for incident valvulopathy among patients [13]. Due to a myriad of potential side-effects, the European Medicines Agency (EMA) has rejected a license to lorcaserin. Among these possible sideeffects are psychiatric disorders, cardiovascular problems and increased risk of cancer.

Anther antiobesity drug ("Qsymia") is a combination of phentermine and topiramate in the form of an extendedrelease medication. Phentermine is known to be useful as short-term appetite suppressant, whereas topiramate is applied as an anticonvulsant drug and is employed to ward off atypical migraine. Phentermine brings about appetite suppression by increasing the concentrations of norepinephrine in the central nervous system. Since topiramate is a $\gamma$ - aminobutyric acid receptor modulator, the acting mechanism of induced weight loss may be through its inhibition of orexigenic glutamate signaling and the increasing of energy depletion. Many trials have tested the efficacy of phentermine and topiramate in weight reduction. Such studies have demonstrated that those patients who were treated with phentermine and topiramate achieved higher weight loss than did placebo-treated patients. In weight loss therapy, Osymia should induce an initial body weight loss of at least $3 \%$ during the course of one prescription. If a patient's body weight percentage does not decline, the patient should stop the use of phentermine/topiramate. What is more, patients undergoing anti-obese treatment with these drugs are prone to struggle with following side effects: paresthesia, dizziness, dysgeusia, insomnia, constipation and dry mouth [9].

Liraglutide $(3 \mathrm{mg})$ is also available for treating weightrelated problems in patients. Liraglutide reduces food consumption and body weight through its dynamic influence on the central nervous system, since it is a glucagon-like peptide-1 (GLP-1) receptor agonist. The drug's agonism influences the central nervous system by way of dynamical input. This increases the level of satiety and reduces food consumptions, hence, bringing about body weight loss [1]. Clinical trials have revealed that a higher dose of liraglutide has led to treatment-related weight loss of $6 \%$ over the administration of a placebo [12]. In such studies, it was also noted that patients with diabetes type 2 were able to achieve significant weight loss, because liraglutide increases insulin resistance and $\beta$-cell function. However, patients should discontinue using the drug when the following side-effects are encountered: nausea, hypoglycemia, diarrhea, vomiting, headache, dyspepsia, fatigue, dizziness, constipation, hypoglycemia, dizziness and diarrhea [1].

Bupropion and naltrexone (marketed as "Contrave" in the USA, and "Mysimba" in Europe) complex therapy was approved by FDA in 2014 as an anti-obesity drug. Naltrexone is an opioid receptor antagonist that reduces cravings by inhibiting the action of $\beta$-endorphins on the dopaminergic reward pathways, whereas Bupropion acts as a norepinephrine and dopamine reuptake inhibitor, and is widely used as an antidepressant drug. The simultaneous combination of these two drugs leads to weight reduction: bupropion stimulates pro-opiomelanocortin (POMC) neurons, while naltrexone counteracts the autoinhibitory effects of the endogenous opioids they secrete [1]. The study known as "Contrave Obesity Research" (COR) was undertaken to test the efficacy of the mentioned complex pharmacotherapy in the treatment for overweight and obese people with BMI $\leq 30-45 \mathrm{~kg} / \mathrm{m}^{2}$ or BMI of $\leq 27 \mathrm{~kg} / \mathrm{m}^{2}$, with dyslipidemia or controlled hypertension. The COR outcome was the following: patients lost $1.3 \%, 5.0 \%$, and $6.1 \%$ of their initial body weight ( $\mathrm{p}<0.0001$ vs. placebo), respectively, and significantly more patients who were on naltrexone-bupropion treatment achieved $\geq 5 \%$ of body weight loss than did those treated with placebo [1]. Constipation, dry mouth, headache, dizziness, vomiting, insomnia, and diarrhea are the most

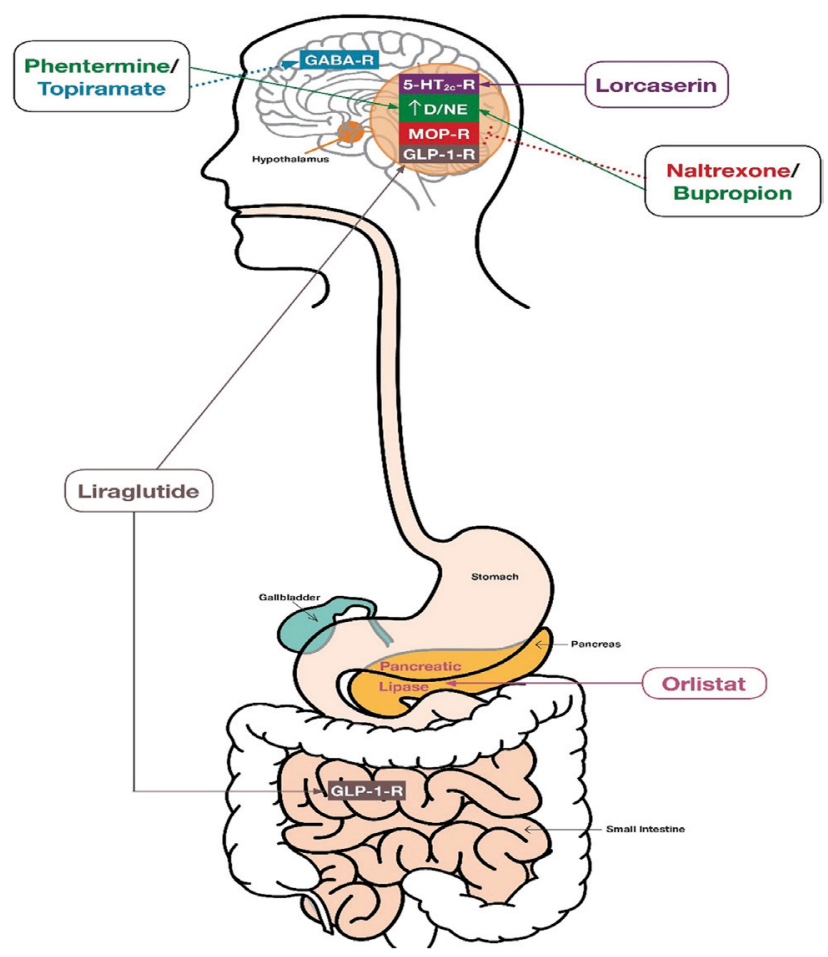

Figure 1. Mechanism of action summary of FDA-approved, longterm anti-obesity pharmacotherapies available by prescription. Dotted arrow indicates the potential mechanism. 5-HT2C-R = 5-hydroxytryptamine (serotonin) $2 \mathrm{C}$ receptor; $\mathrm{D} / \mathrm{NE}=$ dopamine/ norepinephrine; GABA-R $=\gamma$-aminobutyric acid receptor; GLP-1-R = glucagon-like peptide-1 receptor; $\mathrm{MOP}-\mathrm{R}=\mu$-opioid receptor [9] 
common adverse effects. In addition, Naltrexone and bupropion complex pharmacotherapy is contraindicated in patients with seizure disorders, anorexia nervosa or bulimia, and in patients with sudden discontinuation of alcohol and benzodiazepines antiepileptic drugs [9].

\section{INNOVATIVE OBESITY TREATMENT}

Since obesity is genuinely an epidemic of the XXI century, approved pharmacotherapy and those in development will continue to play an important role in combating it and its associated comorbidities. Despite the withdrawal of rimonabant, recent studies targeting the $\mathrm{CB} 1$ receptor have had promising results. In contrast to rimonabant, newer pure CB1 receptor neutral antagonist agents are associated with generating less adverse effects and have demonstrated significant weight loss [3].

Currently, the obese or overweight have access to tesofensine, a monoamine reuptake inhibitor, as a pharmacological drug for maintaining body weight loss. This agent suppresses appetite and increases thermogenesis in the human body [11].

Another drug, velneperit, a neuropeptide Y type 5 receptor (Y5) antagonist, also controls appetite level, doing so by reducing the feeling of hunger. It is considered to be one of the most tolerant anti-obesity drug available today. Of note, this drug may cause the following side effects: sinusitis, upper respiratory infection and headache [11].

Beloranib is a recently approved methionine aminopeptidase 2 inhibitor which reduces lipid biosynthesis and stimulates lipid oxidation/lipolysis in the human body. Patients who have been administered this drug on a daily basis have achieved significant fat reduction [11].

Mirabegron, a $\beta 3$-adrenergic receptor agonist (AR), has also been found to play a role in body weight loss. Hence, it is considered to be a promising and efficient pharmacotherapy for weight reduction. That drug has in vitro binding specificity for the human $\beta 3-\mathrm{AR}$, and it works by relaxing the muscles in the bladder. However, among its side-effects are dizziness, increased blood pressure and blurred vision. Furthermore, it is contraindicated for pregnant women, and those with kidney, liver and cardiac problems [3,11].

\section{BARIATRIC SURGERY}

Beyond pharmacotherapy, when a patient fails to apply this, a healthy diet and physical activity to reduce heft and body mass, there is still another way to lose weight. This is called "bariatric surgery". The aforementioned treatment option means that a patient will eat less after undergoing an invasive procedure during which their digestive system's anatomy will be changed. Yet, not every obese or overweight person may undergo this form of surgery There are currently three major types of bariatric surgery procedures. The first one is called "laparoscopic adjustable gastric banding", wherein a small band is placed around the upper stomach.
The second one is deemed "sleeve gastrectomy", during which a surgeon removes a large part of stomach. The third one is called "gastric bypass surgery". Here, a surgeon directly connects a part of the stomach with the intestine, bypassing a major portion of the stomach and a section of the duodenum [10]. It should be noted that there is currently a lack of evidence for or against recommending bariatric surgery for patients with BMI $<35 \mathrm{~kg} / \mathrm{m}^{2}$. Moreover, as stated previously, not every patient who struggles with weight problems can undergo bariatric surgery [7].

\section{CONCLUSIONS}

In regard to the obesity epidemic problem of the XXI century, pharmacotherapy is an invaluable method in treating weight-related problems. The aforementioned described pharmacological drugs have been thoroughly tested and have been approved for obesity treatment by many agencies of international repute. Indeed, health practitioners and clinicians frequently adopt pharmacotherapy as the underlying solution for their obese and overweight patients.

\section{REFERENCES}

1. Ben J.J., Stephen B.: The new era of drug therapy for obesity: the evidence and the expectations. Drugs, 75, 935, 2015.

2. Bragg R., Crannage E.: Review of pharmacotherapy options for the management of obesity. J. Ann. Assoc. Nurse. Pract., 10,1002/23276924.12279, 2015.

3. Bray G.A., Ryan D.H.: Update on obesity pharmacotherapy. Ann. NY. Acad. Sci., 1311, 1-13, 2014.

4. Główny Urząd Statystyczny: Notatka informacyjna Zdrowie i zachowanie zdrowotne mieszkańców Polski w świetle Europejskiego Ankietowego Badania Zdrowia (EHIS)., 5, 2014.

5. Hannah N.: What is obesity? http://www.medicalnewstoday.com/ info/obesity/, 22.02.2015.

6. Hunt L.E. et al.: Complete re-sequencing of a $2 \mathrm{Mb}$ topological domain encompassing the FTO/IRXB genes identifies a novel obesity-associated region upstream of IRX5. Genome Med., 10,1186/ s13073-015-0250-3, 2015.

7. Jensen M.D. et al.: Guideline for the Management of Overweight and Obesity in Adults: a report of the American College of Cardiology/ American Heart Association Task Force on Practice Guidelines and The Obesity Society. J. Am. Coll. Cardiol., 63, 2985-3023, 2013.

8. Ogden C.L. et al.: Prevalence of childhood and adult obesity in the United States, 2011-2012. JAMA, 311, 806-14, 2014.

9. Patel D.: Pharmacotherapy for the management of obesity. Metabolism, 10, 1016, 2015.

10. Seger J.C. et al.: Obesity Algorithm ${ }^{\oplus}$, http://www.asbp.org/ obesityalgorithm.html, 22.12.2015.

11. Sweeting A.N et al.: Pharmacotherapy for the treatment of obesity. Mol. Cell. Endocrinol., 418, 173-183, 2015.

12. Wadden T.A. et al.: Weight maintenance and additional weight loss with liraglutide after low-calorie-diet-induced weight loss. The SCALE Maintenance randomized study. Int. J. Obes. Lond., 37(11), 1443-51, 2013.

13. Weissman N.J.: Echocardiographic assessment of cardiac valvular regurgitation with lorcaserin from analysis of 3 phase 3 clinical trials. Circ. Cardiovasc. Imaging., 6(4), 560-7, 2013.

14. World Health Organization: Global status report on noncommunicable diseases, 2014, www.apps.who.int/iris/ bitstream/10665/148114/1/9789241564854_eng.pdf?ua=1.

15. Yanovski S.Z., Yanovski J.A.: Long-term drug treatment for obesity: a systematic and clinical review. JAMA., 311,74-86, 2014. 